121 THYMUS IN NORMAL NEWBORN AND IN CYANOTIC HEART DISEASE L. George, F. Elbl and R.E. Solinger, University of Loulsville School of Medicine, Louisville, Ky. 40201 Radiological thymic size was compared in 3 groups of fullterm newborn infants in the first 2 days of life; Group(a) -50 normal (NNBs), Group(b)-39 severely cyanotic congenttal heart disease (SCCHD) with 21 complete D-transposition of great arteries (CTGA) 10 valvular pulmonary atresia/severe stenosis (PA), 5 tricuspid atresia(TA), 3 tetralogy of fallot (TOF) and Group(c) 21 mildiy cyanotic congenital heart disease (MCCHDs) with 8 hypoplastic left heart syndrome (HLH), 13 miscellaneous complex lesions (MCL). Diagnosis in groups (b) and (c) were confirmed by cardiac cathete rization and angiography. Frontal plain chest films were reviewe In all and thymic size was graded (G) as follows: absent-GO; ma11 G1; moderate-G2 and large-G3.

In NNBs $80 \%$ ( 40 and 50 ) revealed G1 to G3 thymus of which $20 \%$ were G3. In SCCHDs only $23 \%$ ( 9 of 39 ) had a thymus, all being G1, with CTGA 28.5\% ( 6 of 21 ), PA 20\% ( 2 of 10), TA $0 \%$ (o of 5 ) and ToF $33 \%$ (1 of 3). In MCCHDs $62 \%$ (13 of 21 ) had thymus of G1/G2 none of G3) with HLH 75\% (6 of 8) and MCL 54\% (7 of 13). Thymus is of ten absent in SCCHDs in comparison to MCCHDs and NNBs in the first 2 days of life. The cardio-resplratory distress which accompanies HLH does not appear to alter thymic size in these infants as compared with NNBs. Level of arterial hypoxemia which dictates the degree of cyanosis, probably is the pertinent stress factor which involutes thymus in infants with cyanotic congenital heart disease. Absence of thymus is not unique to CTGA in the newborn, but also is encountered in other types of SCCHDs.

122 CONCEALED ANOMALOUS CARDIAC CONDUCTION PATHWAYS. A FREQUENT CAUSE OF SUPRAVENTRICULAR TACHYCARDIA. Paul C. Gillette, Baylor College of Medicine \& Texas

Children's Hospital,Department of Pediatrics (Cardiology), Houston Optimal treatment of supraventricular tachycardia (SVT) is aided by a knowledge of its electrophysiologic mechanisms. SVT may be due to an ectopic focus in the atrium or atrioventricular $(A-V)$ junction or reentry (R). Previously, most $R$ circuits in patients without Wolff-Parkinson-White (WPW) syndrome have been thought to be contained in the A-V or sinoatrial nodes. Using His thought to be contained in the A-V or sinoatrial nodes. Using His bunde and multiple atrial recording sites, together with programmed atrial and ventricular stimulation it was possible to
document extra-nodal concealed anomalous conduction pathways (CACP) which participated in $R$ circuits in 10 of 15 patients, 3 weeks to 44 years old, with known SVT without WPW.

Three electrophysiologic findings defined the existance of the CACP, its location, and its participation in the $R$ circuit. 1)Eccentric retrograde atrial activation during SVT: the left atrium was activated first in 6 , the posterior atrial septum in 1 , and the right atrium in 3.2 ) Atrial capture by a premature ventricular stimulus when the bundle of His was refractory. 3) Slowing of the SVT rate and lengthening of V-A conduction coincident with bundle branch block on the side of the CACP. The importance of accurate diagnosis of this form of SVT is shown by the fact that 2 of these patients have had relief of the tachycardia after surgical division of the CACP. Medical management was enhanced al so by these studies by directing therapy to drugs such as propranolol, known to slow conduction in anomalous pathways.

RESULTS OF ORAL PROPRANOLOL TREATMENT IN INFANTS AND CHILDREN. Paul C. Gillette, Erna Eterovic, William Neches, Charles E. Mullins, and Dan G. McNamara, Baylor College of Medicine and Texas Children's Hospital, Dept. of Pediatrics (Cardiology). Houston.

Propranolol has been extensively studied in adults but its use in children has been infrequently reported. Fifty infants and children treated with ora! propranolol are presented. In 34 subjects, an investigational liquid form of the drug was used. The diagnoses were: 1) tachydysrhythmias $(27), 2)$ decreased pulmonary blood flow and hypoxemic spells (17) and 3) idiopathic hypertrophic subaortic stenosis (IHSS) (6). In each group, effectiveness, dose used, and side effects, including postoperative problems, were analyzed.

In the patients with decreased pulmonary flow and "spells."

In the patients with decreased pulmonary flow and "spells,
propranolol treatment was effective in $76.4 \%$ (13 of 17). Tachypropranolol treatment was effective in $76.4 \%$ (13 of 17). Tachydysrhythmias were abolished in $62.9 \%$ (17 of 27). Propranolol relieved the symptoms in each of the 6 patients with IHSS. Use of
the 1 iquid form enhanced treatment in infants by case of administration and the ability to make small changes in doses. The liquid form was found to be equally as effective as the tablet. side effects to propranolol included bradycardia in 4 patients. Side effects to propranolol included bastive heart failure in 1 and wheezing in 1 . Postoperative low cardiac output was not observed.

Based on this study, we conclude that propranolol is an effective and safe drug for use in pediatric patients with certain types of cardiac disease.
124 MIOCARDIAL HYPOXIA DURING ISOPROTERENOL INFUSION IN AN ANIMAL MODEL: POSSIBLE CAUSE OF MYOCARDIAL DYS

Thomas P. Graham, Glenn S. Buckspan, R. Darryl Flsher, Vanderbilt University. Dept of Pediatrics. Nashville, Tennessee Myocardial dysfunction has been reported in cyanotic congenital heart disease (CCHD) patients. One possible cause of this abnormality could be recurrent eplsodes of myocardial hypoxia during mality could be recurrent eplsodes of myocardial hypoxia during
periods of high myocardlal $\mathrm{O}_{2}$ demand. Accordingly an animal model was developed to produce systemic hypoxemia with a tefion graft used to shunt the infertor vena caval return to the left atrium. Left ventricular pressure (LVP), dP/dt, aortlc pressure, heart rate (HR), $\mathrm{PO}_{2}, \mathrm{ph}, \mathrm{PCO}_{2}$, and arterial (a) and coronary sinus (cB) lactate (LAC) levels were measured before and after atrial pacing (AP) and isoproterenol (ISU) infusion in shunted and control dogs. In four control animals ( $\mathrm{pO}_{2} 39-58 \mathrm{mmHg}$ ) suband control dogs. In four control animals $\left(\mathrm{pO}_{2} 39-58 \mathrm{mmHg}\right.$ ) sub-
jected to AP, HR increased from $\frac{147}{233} / \mathrm{min}$ while $\mathrm{LAC}$ (a-c8) jected to AP, HR increased from 147 to $233 / \mathrm{m} / \mathrm{n}$ while LAC (a-c8)
did not change significantly $(0.9$ to $1.4 \mathrm{meq} / \mathrm{L})$; with ISU there did not change significantly $(0.9$ to $1.4 \mathrm{meq} / \mathrm{L})$; with ISU there
was also no change in LAC $(0,9 \mathrm{meq} / \mathrm{L})$ desplte an increase in HR and $\mathrm{dP} / \mathrm{dt}$ from control. Five cyanotic animals ( $\left.\mathrm{pO}_{2} 24-30 \mathrm{mmHg}\right)$ also showed no significant change in LAC (a-cs) with AP $(\overline{0.9}$ to $1.1 \mathrm{meq} /$ L) but all dogs showed lactate production ( $\mathrm{LAC}(\mathrm{a}-\mathrm{Cs})=-0.6 \mathrm{meq} / \mathrm{L}$ ) with ISU infusion. These data indicate that myocardial hypoxia can be produced in a cyanotic animal model during times of high myucardial $\mathrm{O}_{2}$ demands at $\mathrm{pO}_{2}$ levels commonly seen in patients with CCHD. Thus myocardial hypoxia may play a role in the ventricular dysfunction seen in children with CCHD.

125 ECHOCARDIOGRAPHIC PITFALLS IN THE PREMATURE WITH LARGE PATENT DUCTUS ARTERIOSUS. By Michael J.
Hirschklau, Stanley E. Kirkpatrick, Charles B. Higgins and William F. Friedman. Div. of Ped. Cardio1., Univ. of Calif., San Diego, School of Medicine. mensions (LA/AO) is used commonly to assess the presence and magnitude of left to right shunting through the PDA in prematures. The results of the present study emphasize the hazards of overreliance on this echo index. Thus, seven of thirty-one consecutive premature infants with severe respiratory distress syndrome and clinical and radiographic evidence of significant ductal left to right shunting were found to have normal LA/Ao ratios, as well as normal absolute left atrial dimensions. The LA/Ao ratios remained normal in these respirator-bound infants despite clinical signs of severe cardiac decompensation. In four of these infants the PDA was ligated surgically, while in two indomethacin was effective. The remaining infant responded to digitalis, diuretics, and tive. The remaining infant responded to digitalis, diuretics, and
fluid restriction, and ultimately closed her PDA spontaneousiy. Abolition of ductal left to right shunting did not consistently change the LA/AO ratios or absolute left atrial dimensions in this group. A major finding in these infants was the detection of abnormally enlarged left ventricular end diastolic dimensions in the presence of normal left atrial dimensions. Thus, maximum diagnostic accuracy can be achieved only by a comprehensive echocardiographic evaluation that combines analysis of both atrial and ventricular dimensions since the former, when used alone, may be misleading.

\section{POLYCYTHEMIA, CARDIAC OUTPUT AND CORONARY BLOOD FLOW.} Julien I.E. Hoffman, Adrianta Surjadhana, Lawrence E. School of Medicine, Department of Pediatrics and Cardiovascular Research Institute, San Francisco.

Decreased cardiac output $(\mathrm{CO})$ and systemic oxygen transport (SOT) in polycythemia (P) are attributed to increased blood viscosity and regarded as potentially harmful. Since $P$ is common cosity and regarded as potentially harmful. Since $P$ is common
in cyanotic heart disease, we studied its effects on CO. SOT and left ventricular coronary blood flow (CBF) and oxygen transport (LVOT) in dogs with acute isovolumic $P$. Hematocrit increase to $65-70 \%$ caused falls in CO and SOT; despite $P$, CO rose with hypoxemia or adenosine infusion. $P$ markedly decreased CBF and cardiac work; slightly decreased LVOT, myocardial oxygen consumption and coronary sinus oxygen saturation; and increased coronary vascular resistance(Rc). Cardiac stress (pacing, aortic stenosis, arteriovenous fistula), hypoxemia and adenosine infusion lowered Rc. Changes in LVOT were more closely related to changes in pressure work and myocardial oxygen consumption than to hematocrit. With maximal coronary vasodilatation, Rc doubled when hematocrit rose from $45 \%$ to $65 \%$. Our results indicate that autoregulation is more important than viscosity in regulating $\mathrm{CO}$, CBF, SOT and LVOT in $P$. With maximal coronary vasodilatation, however, increased blood viscosity in $P$ reduces LVOT and suggests that with maximal stress there could be ischemic myocardiai damage. 